Supporting Information

\title{
Elastic-Plastic Deformation of a Solid Electrolyte Interface Formed by Reduction of Fluoroethylene Carbonate: A Nanoindentation and Finite Element Analysis Study
}

Yuki Kamikawa, $, \#, \#, *$ Kouji Amezawa, ${ }^{\ddagger}$ and Kenjiro Terada ${ }^{\mathcal{S}}$

‡Institute of Multidisciplinary Research for Advanced Materials, Tohoku University, 21-1 Katahira, Aoba-ku, Sendai 980-8577, Japan

§International Research Institute of Disaster Science, Tohoku University, 468-1-S403, Aoba, Aramaki, Aoba-ku, Sendai 980-0845, Japan

\#Nissan Motor Co., Ltd. 1-1-1, Nishi-ku, Yokohama 220-8686, Japan

${ }^{\dagger}$ Research Center, Envision AESC Japan Ltd., 4-2-11 Hironodai, Zama 252-0012, Japan 


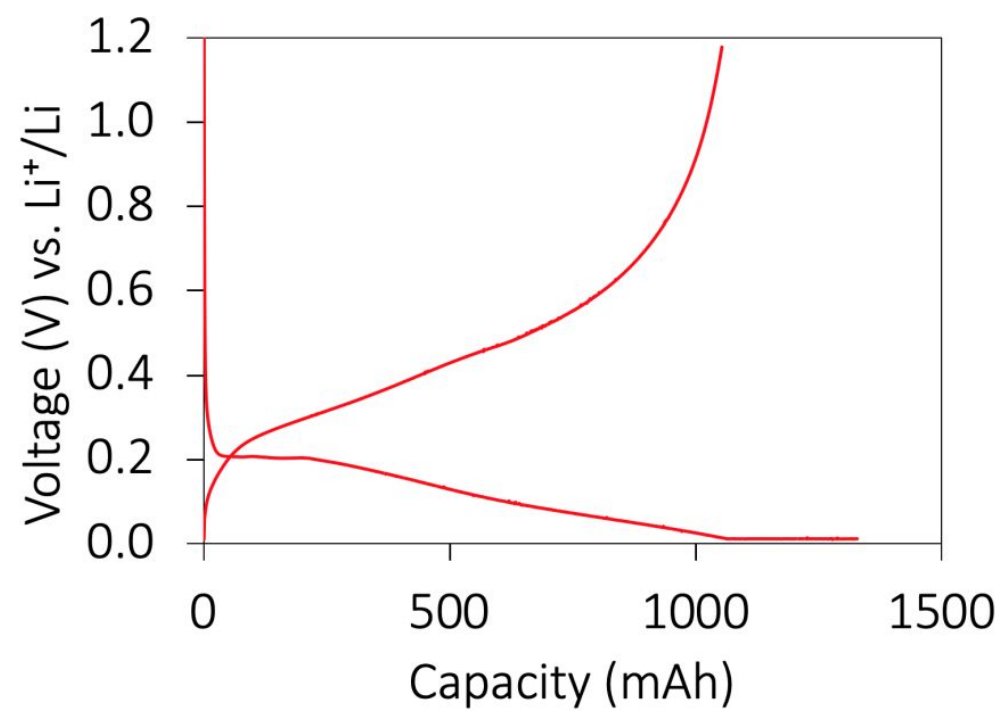

Figure S1. Charge-discharge curves of SiSnTi electrode in $1 \mathrm{M} \mathrm{LiPF}_{6} / \mathrm{EC}-\mathrm{FEC}$ electrolyte.
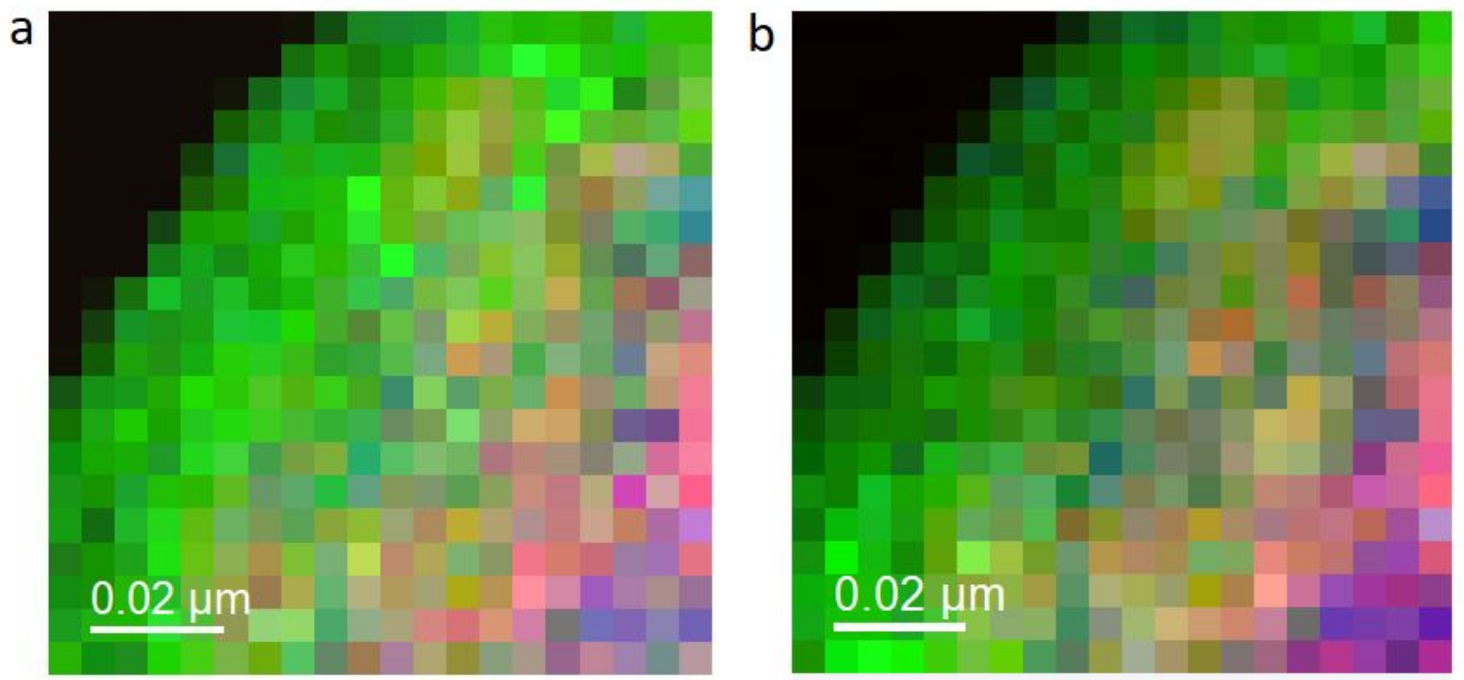

Figure S2. EELS mappings of (a) O (green), (b) Li (green), Si (blue), and Ti (red) obtained for the cross-section of SEI and SiSnTi. 


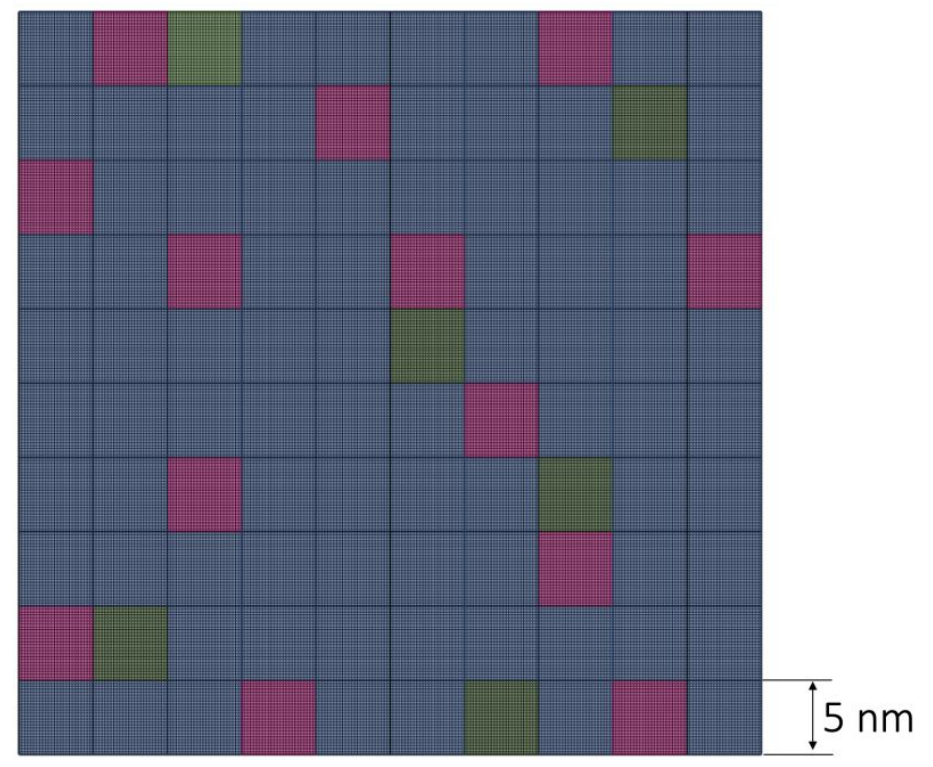

Figure S3. Meshing of the SiSnTi model along with the distributions of Si (blue), Sn (green), and Ti (red) used for FEA simulation. 